\title{
¿CONSULTA O ASESORAMIENTO? ANÁLISIS DEL USO DE ESTOS DOS TÉRMINOS ENTRE LOS PROFESIONALES DE LA ORIENTACIÓN
}

\author{
Nuria Pérez-Escoda ${ }^{1}$ \\ Rafael Bisquerra Alzina \\ Universidad de Barcelona
}

CONSULTATION OR COUNSELING?

\section{RESUMEN}

Asesoramiento y consulta son dos términos que se usan en orientación para referirse al mismo concepto. Este artículo pretende aportar elementos de reflexión para su uso apropiado. El término consulta (consultation) tiene una larga historia en orientación. Se inicia en los años sesenta y con el tiempo se ha ido configurando como el modelo básico por excelencia en orientación. La expresión y el concepto responde a consulta colaborativa. Sin embargo, en el lenguaje más habitual de la mayoría de los profesionales de la orientación se utiliza asesoramiento para referirse prácticamente a lo mismo. Esta doble terminología a veces produce vacilaciones en el uso del lenguaje, de tal forma que hay autores que utilizan los dos para evitar confusiones (consulta-asesoramiento), lo cual es un claro reflejo de la imprecisión terminológica que se utiliza. Este trabajo analiza el estado de la cuestión con el propósito de aportar evidencias que permitan tomar decisiones con conocimiento de causa y poder avanzar hacia una terminología unívoca, propia de la metodología científica. Para ello se revisa la frecuencia en el uso de las palabras consulta y asesoramiento en la bibliografía y seguidamente, se aplica un cuestionario a una muestra de 228 profesionales especialistas en orientación para conocer el término que están utilizando. Los resultados ponen de manifiesto que si bien en la terminología científica en inglés se utiliza consultation, en el uso entre los profesionales en España es preponderante el uso de asesoramiento.

\footnotetext{
1 Correspondencia: Universidad de Barcelona. Departamento MIDE, Despacho 208 Campus Mundet -Edifici Llevan Pg. Vall d'Hebrón, 17108035 Barcelona Correo-e: nperezescoda@ub.edu
} 
Palabras clave: consulta, asesoramiento, consulta colaborativa, orientación psicopedagógica, modelos de orientación.

\section{ABSTRACT}

In Spanish language there are two words used in the same sense in the educational guidance field: "Asesoramiento" and consultation are two words used in same sense. This paper has the intention to bring arguments for the correct use of each one of them. The term consultation has a long history in educational guidance. It begins in the sixties and over time has became the basic model in educational guidance. The term and concept responds to collaborative consulting. However, in the language of most practitioners of guidance and counseling in Spain use "asesoramiento" to refer to the same concept. This double terminology sometimes causes hesitation, and authors use the two of them indistinctly to avoid confusion ("consulta""asesoramiento" in Spanish), which is a clear reflection of the imprecision of terminology used. This paper analyzes the status quaestionis using a questionnaire applied to 228 specialists. The evidence is that in specialized literature the term used is consultation; nevertheless the professionals in Spain mainly use "asesoramiento". This evidence may help to take decisions.

Keywords: Consultation, counseling, collaborative consultation, guidance, counseling models.

\section{Introducción}

Este artículo tiene por objetivo analizar el uso de dos términos que a veces se usan como sinónimos: consulta y asesoramiento. En cualquier rama de la ciencia se utiliza una terminología específica y existe un acuerdo sobre el uso y el significado de lenguaje especializado. Esto no siempre pasa en orientación y consideramos necesario consensuar el uso de los términos para darles el mismo significado que sea asumido para la toda la comunidad científica y de los profesionales de la orientación.

A tal efecto, se presenta el concepto de consulta en la tradición americana y en Europa, con especial referencia a España. Se analiza como se ha utilizado asesoramiento en castellano como sinónimo de consulta, lo cual a veces ha producido ciertas confusiones. En el fondo hay dos tradiciones distintas con lenguajes distintos.

A partir de esta situación se ha aplicado una encuesta para conocer la terminología más utilizada por los expertos en orientación. Se presentan los datos obtenidos con unas reflexiones y propuestas para la práctica con vistas a una posible unificación terminológica.

\section{El uso de la consulta en orientación}

En las publicaciones especializadas en inglés se habla de consultation para referirse a una de las funciones más importantes de la orientación, que consiste en una intervención indirecta en la cual un profesional de la orientación colabora con el profesorado (puede ser uno o varios) para que sea éste el que intervenga directamente con el alumnado, siguiendo una serie de pautas expuestas en la literatura especializada. El destinatario final siempre es el alumnado. Aunque para ello se puedan realizar diversas acciones dirigidas al profesorado, a la institución, a la familia o a la sociedad. 
El modelo de consulta propone que el orientador dedique el máximo de tiempo a trabajar de forma colaborativa con el profesorado y con la institución educativa en lugar de intervenir directamente con el alumnado. Se trata de una intervención indirecta. Esto supone reducir al mínimo el modelo clínico y potenciar el modelo de consulta y de programas.

Como mínimo desde principios de los años sesenta se está utilizando en la literatura especializada en orientación en inglés la palabra consultation. Algunos ejemplos de los primeros artículos en este sentido son: The Consultant in Elementary School Guidance (Dinkmeyer, 1962), The Counselor as a Consultant to Teachers (Faust, 1967), Is a Guidance Consultant Needed in the Elementary School? (Lee, 1963), etc.

A partir de los años setenta se observa un aumento de publicaciones que contribuyeron a difundir el modelo de consulta en orientación. Algunos ejemplos ponen de manifiesto que en esta década se empiezan a crear programas de formación de consultores, como por ejemplo el de Gallessich y Ladogana (1978), Consultation Training Program for School Counselors; Kurpius y Brubaker (1976) hablan de Psychoeducational consultation; Meyers (1978) se refieren a la importancia de formar al psicólogo escolar para el rol de consultor; Young y Borgen (1979) aplican la consulta para la implementación de programas de educación para la carrera; Brown et al. (1979) utilizan la consulta como estrategia para mejorar la educación.

En la bibliografía se pueden encontrar muchos artículos que ponen de manifiesto como la consulta en orientación no es algo novedoso ni marginal. Sino que se utiliza desde hace décadas y que constituye uno de los modelos esenciales de la orientación (Aubrey, 1990). La consulta en orientación suele adoptar la forma de consulta colaborativa. Pryzwansky (1977) es uno de los pioneros de la consulta colaborativa. No es casualidad que West (1990) le hiciera una entrevista que se publicó con el título de The nature of consultation vs. colaboration: An interview with Walter B. Pryzwansky, donde se hace referencia al tema de la consulta colaborativa, que es distinta de la consulta de experto.

En la consulta colaborativa se asume una responsabilidad compartida entre consultor y consultante: ambos analizan el contexto conjuntamente, se ponen de acuerdo en los objetivos, desarrollan un plan de acción, se proponen ponerlo en práctica y evaluarlo de forma colaborativa.

West e Idol (1987) serán continuadores de Pryzwansky. Estos autores informan que entre 1978 y 1983 se publicaron casi un millar de libros y artículos sobre consulta colaborativa. Idol, Paolucci-Whitcomb y Nevin (1986) profundizan en el tema; West y Cannon (1988) se ocupan de las competencias necesarias para aplicar este modelo; Idol (1990) se refiere al arte científico de la consulta, etc. Otros autores también se han ocupado de la consulta colaborativa, como por ejemplo Robinson (1991), Erchul y Martens (1997), Schulte y Osborne (2003).

En 1990 se crea el Journal of Educational and Psychological Consultation (JEPC) que proporciona un forum para mejorar la comprensión científica de la consulta y para la descripción de las estrategias prácticas para aumentar la eficacia y eficiencia de los servicios de consulta en educación y en psicología. En esta revista se define consulta como un proceso que facilita la resolución de problemas para los individuos, grupos y organizaciones.

A la luz de lo expuesto, es evidente que en el contexto americano existe una larga tradición en el uso de la consulta, y en concreto de la consulta colaborativa en educación y orientación, que justifica el uso de este concepto como un modelo básico de la práctica de la orientación.

Con la relevancia que toma la consulta en el contexto americano, no es extraño que llegue a Europa y Latinoamérica, como una propuesta de innovación que afecta a las funciones 
tradicionales de los profesionales de la orientación.

De esta forma, en los años setenta se publica en Buenos Aires la traducción al castellano de la obra de Dinkmeyer y Carlson (1973) con el título de El consultor psicopedagógico en la escuela. La traducción de consultant por consultor parece la más lógica.

También en francés se utiliza la palabra consultation, como mínimo desde la década de los años setenta. Así, por ejemplo, Lamothe (1976) habla de Les professionnels de la consultation et de l'orientation en milieu scolaire: des intervenents en mutation, publicado en la revista L'orientation professionnelle.

En consonancia con esta tendencia, desde los años ochenta se ha estado hablando de consulta en la formación de los orientadores, como mínimo en varias universidades europeas y españolas.

Como era de esperar, diversos autores han contribuido a difundir en castellano el modelo de consulta en orientación, entre los cuales cabe citar a título de ejemplo a Rodríguez Espinar (1986, 1993), Bisquerra (1990, 1996, 1998), Bisquerra y Álvarez $(1998,2000)$, Santana Vega y Santana Bonilla (1998), Sanz Oró (2001: 137-159), Martínez Clares (2002) y un largo etcétera.

\section{De la consulta al asesoramiento}

A pesar de todos los argumentos anteriores y citas bibliográficas de larga tradición en orientación, se observa una tendencia en utilizar en castellano asesoramiento en lugar de consulta. Pueden encontrarse muchas publicaciones en castellano donde se utiliza asesoramiento con el mismo significado que se le da a consulta en las publicaciones especializadas en inglés. Algunos ejemplos son los siguientes: El asesoramiento psicopedagógico: una perspectiva constructivista (Bassedas, 1988); El asesoramiento a centros educativos (Escudero y Moreno, 1992); El asesoramiento en educación (Rodríguez Romero, 1996); El asesoramiento psicopedagógico: una perspectiva profesional y constructivista (Monereo, y Solé, 1996); Los equipos de orientación educativa y psicopedagógica. El asesoramiento a centros escolares desde un análisis institucional (Nieto y Botías, 2000); Asesoramiento al centro educativo (Domingo, 2001); Manual de asesoramiento psicopedagógico (Bonals y Sánchez-Cano 2007); Asesoramiento psicopedagógico y mejora de la práctica educativa (Lago y Onrubia, 2008; 2011); La práctica del asesoramiento educativo a examen (Monereo y Pozo, 2005), etc.

La lectura de los títulos de estas obras permite observar el significado y la tendencia otorgada al concepto de asesoramiento psicopedagógico. Sería fácil pensar que asesoramiento y consulta son cosas distintas. Con la intención de que no se confundan los estudiantes y lectores poco habituados a estos términos conviene dejar claro que, cuando en orientación se habla de asesoramiento, se está hablando de lo mismo que consultation en inglés.

El uso indistinto de esta doble terminología produce redactados curiosos, que no hacen sino poner de manifiesto la confusión terminológica. Así, por ejemplo, en Santana (1998: 60) se puede leer: "West \& Idol (1987) citan cinco ámbitos desde los que se realizan labores de asesoramiento..." Cuando se consulta la bibliografía se descubre que West e Idol, en realidad se refieren a la school consultation.

En el Manual de asesoramiento psicopedagógico, coordinado por Bonals y Sánchez-Cano (2007: 91), se puede leer: "Lorna Idol (1990) expone en un artículo que lleva el sugerente título de El arte científico del asesoramiento en las aulas" (p. 91). Cuando vamos a consultar la referencia 
original se observa que el título en realidad es: The scientific art of classroom consultation, publicado, precisamente, en el Journal of Educational Psychology Consultation.

En el libro Orientación educativa. Modelos y estrategias de intervención, coordinado por Martín y Solé (2011), se puede leer: “... es a lo que suele denominarse asesoramiento (véase la revisión de West e Idol, 1987)". Esta cita se refiere a unos autores reconocidos en el campo de la school consultation, que nunca han utilizado "asesoramiento".

Los ejemplos podrían seguir. En las traducciones a veces se pone consulta y otras asesoramiento. Curiosamente, en general, en los trabajos en que se habla se asesoramiento, contrasta la abundancia de citas a autores en castellano y la escasez de citas a textos en inglés. Mientras que en los textos en que se habla de consulta, el contraste tiende a ser a la inversa: muchas referencias en inglés junto a pocas en castellano. La interpretación de este hecho es lógica: el autor que consulta textos en castellano, se encuentra más frecuentemente el término asesoramiento, y utiliza esta palabra; en cambio el autor que consulta muchos textos en inglés, se encuentra con consultation y tiende a traducirlo por consulta.

Dando un paso más en la interpretación de este hecho, se puede inferir que en general se consulta poco en inglés y mucho en castellano. Lo cual pone en cuestión que las revistas de verdadero impacto sean en inglés, como mínimo en el campo de la orientación.

\section{Asesoramiento y counseling}

Para acabar de complicar la torre de Babel de la orientación, conviene tener presente que la palabra counseling, que es el referente de la orientación en inglés, no tiene una traducción clara en castellano. Por esta razón muchas veces se tiende a no traducirla, dejándola en inglés. Pero otras veces sí que se ha traducido. En estos casos, lo habitual ha sido traducir counseling por asesoramiento. Véase por ejemplo el clásico de Shertzer y Stone (1972), Manual para el asesoramiento psicológico (Counseling), que tuvo una gran influencia en su momento, y que todavía se usa y se cita (véase por ejemplo Santana Vega, 2003). Otros autores, como por ejemplo Sanz Oro (2001: 28) utilizan asesoramiento como traducción de counseling.

El sentido que aquí se le da a asesoramiento equivale a modelo clínico y por lo tanto tiene un significado diametralmente opuesto a consulta. Con lo cual, los lectores que no sean expertos en estos vericuetos del lenguaje, como por ejemplo los estudiantes, pueden quedar confundidos.

Ante estos hechos, la pregunta es ¿en el campo de la orientación, hablamos de consulta o de asesoramiento? La decisión la van a tomar los profesionales. Pero consideramos que sería conveniente avanzar hacia una terminología unívoca que evite duplicidades y confusiones.

\section{Búsqueda bibliográfica}

Conviene tener presente que en el amplio marco de la orientación se utilizan multitud de conceptos que no siempre son utilizados con el mismo significado. Por esto, de vez en cuando puede ser apropiado reflexionar para delimitar y concretar estos conceptos. Es lo que nos proponemos aquí. La pregunta que nos formulamos es sobre la frecuencia en el uso de consulta 0 asesoramiento.

Para conocer el estado de la cuestión en nuestro contexto, se han realizado una búsqueda en las tres revistas españolas de mayor relevancia para los profesionales de la orientación: REOP (Revista Española de Orientación y Psicopedagogía), RELIEVE (Revista Electrónica de 
Investigación y Evaluación Educativa) y Revista de Investigación Educativa (RIE)

En el proceso de búsqueda se han utilizado las siguientes palabras clave: "Asesoramiento", "Asesoramiento psicopedagógico", "Asesoramiento y orientación", "Consulta psicopedagógica", "Consulta y orientación", "Consulta", "Consulta colaborativa", "Asesoramiento and orientación", y "Consulta and orientación".

Esta búsqueda se ha realizado en octubre de 2012, obteniendo los resultados que se resumen a continuación. En la REOP se han hallado 105 títulos con "asesoramiento" por 59 con "consulta". En la RIE, entre 1983 y 2012, se produce un empate con 5 en cada caso. Por lo que respecta a una búsqueda en la web de RELIEVE se han encontrado 25.700 veces "consulta" por 4.710 de "asesoramiento". En cuando a RELIEVE se supone que la palabra "consulta" es utilizada en sentidos muy distintos. Por lo tanto, lo más probable es que solamente la REOP aporte datos que se ajusten al uso real de estos términos en el contexto profesional de la orientación.

Esto pone de relieve la situación de diversidad terminológica.. Si consideramos que la terminología unívoca es un aspecto importante para la construcción de una ciencia, entonces hemos de concluir que tal vez merezca la pena ponernos de acuerdo. Sin embargo la decisión no es fácil.

\section{Método}

\section{La opinión de los profesionales de la orientación}

Hemos considerado oportuno realizar un sondeo sobre el uso de las palabras consulta y asesoramiento entre los profesionales de la orientación. A continuación se expone el procedimiento y los resultados obtenidos. Para recoger de primera mano la opinión de los expertos y profesionales de la orientación de nuestro país se ha utilizado la técnica de encuesta a través de la aplicación de un cuestionario.

\section{El cuestionario}

El instrumento utilizado ha sido un cuestionario que se puede consultar en: https://docs.google.com/spreadsheet/viewform?formkey=dHo4TIQtR2ZyU1B0UVRRczBYOHJGN VE6MQ

En este cuestionario se formulan una serie de preguntas con el objetivo de recoger información sobre el uso de ambos conceptos en la práctica. Al mismo tiempo se formulan otras cuestiones para poder tener más información. El reto era hacer un cuestionario lo suficientemente breve para que al poder ser respondido en unos pocos minutos se obtuviese una muestra grande. El formato del cuestionario es el siguiente. 


\section{Cuestionario sobre "consulta o asesoramiento"}

Este cuestionario tiene el objetivo de contribuir a una clarificación terminológica en el campo de la orientación. Tus respuestas son muy importantes.

\section{Datos identificativos:}

Sexo:

Edad:

Nivel formativo (señalar solamente el más alto):

Diplomado: Licenciado-Graduado Doctor ¿En qué Universidad? *:

Titulación académica (se puede marcar más de una):

Maestro/a

Educación Social

Pedagogía

Psicología

Psicopedagogía

Otros: ¿Cuál?*

¿Trabajas o has trabajado en tareas relacionadas con la orientación?: Sí No

En caso afirmativo, ¿dónde?

Profesor de universidad

Orientación en un centro educativo

Orientación en equipos de sector (EOEP, EAP, etc.)

Orientación en servicios públicos de empleo

Empresas privadas de orientación

Otros: ¿Cuáles? *

Años de experiencia laboral en el campo de la orientación:

Una de las funciones más importantes de la orientación consiste en una intervención indirecta en la cual un profesional de la orientación colabora con el profesorado (puede ser uno o varios) para que sea éste el que intervenga directamente con el alumnado, siguiendo una serie de pautas negociadas y consensuadas. El destinatario final siempre es el alumnado. Aunque para ello se puedan realizar diversas acciones dirigidas al profesorado, a la institución, a la familia o a la sociedad. El orientador dedica el máximo de tiempo a trabajar de forma colaborativa con el profesorado y con la institución educativa en lugar de intervenir directamente con el alumnado.

¿Has aplicado esta estrategia?: Sí $\quad$ No ¿Cómo denominas a esta estrategia?:

Consulta

Asesoramiento

Otras: ¿Cuáles?

¿Cómo crees que se debería denominar? 


\section{Consulta \\ Asesoramiento \\ Otras ¿Cuáles?}

¿Qué escuchas o lees con más frecuencia?

Consulta

Asesoramiento

Otras: ¿Cuáles?

¿Cuántos libros sobre orientación en castellano has consultado en el último año?

¿Cuántos artículos en revistas especializadas en orientación en castellano has consultado en el último año?

¿Cuántos libros sobre orientación en ingles has consultado en el último año?

¿Cuántos artículos en revistas especializadas en orientación en inglés has consultado en el último año?

Si deseas añadir alguna información o algún comentario puedes hacerlo aquí:

Te rogamos escribir el DNI que será utilizado con absoluta confidencialidad y solamente a efectos de evitar dobles envíos de este cuestionario.

DNI:

\section{Muchas gracias por tu colaboración}

Te agradeceremos que reenvíes este cuestionario a otros profesionales de la orientación.

\section{Procedimiento}

El cuestionario se ha difundido a través de un mailing masivo a través de asociaciones de profesionales, redes, foros, Universidades, etc. Consideramos que se han utilizado los recursos disponibles para que el cuestionario haya llegado a todas las asociaciones de profesionales de orientación de España, la COPOE (Confederación de Organizaciones de Psicopedagogía y Orientación de España), AIDIPE, FEOP, la Red Interuniversitaria de Profesorado de Orientación, etc. En el correo, para invitar a responder, se explicaba el objetivo del sondeo y se rogaba dar respuesta al cuestionario on-line al que se accede mediante el enlace anterior.

\section{Descripción de la muestra de participantes}

Ha respondido al cuestionario una muestra de 228 participantes, distribuidos por especialidad (pedagogía, psicología, psicopedagogía, otros) y por titulación (diplomado, licenciado, doctor) de acuerdo a la Tabla 1. Como se puede observar, está equilibrada la muestra de pedagogía y psicología, sobretodo en la titulación de licenciatura. 
TABLA 1. Muestra: titulación por especialidad

\begin{tabular}{|l|c|c|c|c|c|}
\hline & Pedagogía & Psicología & Psicopedagogía & Otros & Total \\
\hline Doctor & 28 & 8 & 9 & 0 & $\mathbf{4 5}$ \\
\hline Licenciado* & 63 & 71 & 36 & 7 & $\mathbf{1 7 7}$ \\
\hline Diplomado & 0 & 0 & 0 & 6 & 6 \\
\hline Total & 91 & 79 & 45 & 13 & $\mathbf{2 2 8}$ \\
\hline \multicolumn{7}{r}{} \\
\hline
\end{tabular}

Respecto a personas con el título de doctor hay muchas más de pedagogía (28) que de psicología (8), como es lógico de esperar por las características de acceso a la muestra. Los 6 diplomados son de Educación Social, Trabajo Social, Maestro, Graduado Social y Relaciones Laborales (2). Además de éstos, hay 7 más que corresponden a la categoría de Otras, cuyas especialidades son: Educación Social (3), Maestro y Derecho (3).

Por lo que respecta al género, hay 75 hombres (32'9 \%) y 153 mujeres (67'1 \%). Esta distribución se corresponde aproximadamente con la relación que hay en el mundo profesional entre los dos géneros.

Por lo que respecta a la edad, la media es de 45’01, con una desviación típica de 9'58. El más joven tiene 25 años y el mayor 70 .

Los participantes de la muestra han estudiado en 36 Universidades distintas. La Universidad Complutense de Madrid es la que tiene una frecuencia más alta con 32 casos. Otras Universidades con un número alto de casos son las de Barcelona, Granada, Salamanca, Santiago, UNED, Sevilla, Málaga, Murcia, etc.

Todos los 228 participantes de la muestra declaran que sí tienen experiencia en orientación. El lugar de trabajo varía considerablemente, siendo las frecuencias más altas para: orientación en un centro educativo, profesor de universidad, orientación en un equipo de sector (EOEP, EAP, etc.), orientación en servicios públicos de empleo, empresas privadas de orientación, etc.

Los años de experiencia profesional presentan una media de 12'42, con una desviación típica de 8'87. El mínimo es de 1 año y el máximo contabilizado es de 40 . Se da el caso de que una persona ha respondido 52 años de experiencia, pero no se ha contabilizado por considerarla outlier.

En su experiencia profesional, hay 187 (82\%) que declaran haber aplicado el modelo de asesoramiento o consulta colaborativa. Los 41 restantes (18\%) declaran que no.

Con los datos aportados, hay elementos para considerar que se trata de una muestra que reúne las características de representatividad para el tema que nos ocupa.

\section{Resultados}

Recordemos que las tres preguntas claves son: a) Denomina: ¿Cómo denominas a esta estrategia?; b) Denominaría: ¿Cómo crees que se debería denominar?; c) Escucha o lee: ¿Qué escuchas o lees con más frecuencia? (Consulta - Asesoramiento). Los resultados obtenidos se detallan en la tabla 2. 
TABLA 2. Uso de asesoramiento o consulta

\begin{tabular}{|l|c|c|c|}
\hline & Denomina & Denominaría & Escucha o lee \\
\hline Asesoramiento & $194\left(85^{\prime} 08 \%\right)$ & $193\left(84^{\prime} 64 \%\right)$ & $173\left(75^{\prime} 87 \%\right)$ \\
\hline Consulta & $27\left(11^{\prime} 84 \%\right)$ & $27\left(11^{\prime} 84 \%\right)$ & $39\left(17^{\prime} 10 \%\right)$ \\
\hline Otros & $7\left(3^{\prime} 07 \%\right)$ & $8\left(3^{\prime} 50 \%\right)$ & $16\left(7^{\prime} 02 \%\right)$ \\
\hline
\end{tabular}

Hay 194 participantes que utilizan asesoramiento frente a solamente 27 que utilizan consulta. Al aplicar la prueba de ji-cuadrado, la $p$ es significativa en todas las posibles combinaciones entre variables (denomina, denominaría, escucha o lee). Queda claro que la inmensa mayoría se inclina por asesoramiento, en detrimento de consulta. Esta información se puede tomar en consideración en el momento de decidir cuál de los dos términos a utilizar.

\section{Análisis discriminante y perfil}

Los datos de la tabla 2 son tan elocuentes que ya dejan claro que hay una gran coherencia entre "denomina" y "denominaría". Al analizar estos datos en una tabla de contingencia solamente hay dos casos en que las respuestas son diferentes en estas dos respuestas. Lo cual nos permite tomar una de las dos (denomina) para los análisis siguientes.

Se han elegido una serie de variables que hipotéticamente podrían presentar parámetros distintos entre el grupo que utiliza asesoramiento respecto al que utiliza consulta: edad, años de experiencia, número de libros consultados, número de artículos consultados, libros en inglés, artículos en inglés. Al aplicar la prueba t de Student para comparas las medias de estas variables entre los grupos que usan "asesoramiento" y "consulta", solamente se observan diferencias estadísticamente significativas en "libros en inglés" ( $p=.044)$ y en artículos en inglés $(p<.000)$. Dicho de otra forma, las personas que usan "consulta" leen más libros y artículos en inglés. Este resultado es coherente con lo que estamos analizando.

Si nos centramos en la especialidad (pedagogía, psicopedagogía y psicología), se puede considerar que las dos primeras pertenecen a educación (ya que se estudia en Facultades de Educación) respecto a la tercera que, en general, es en Facultades Psicología. Es decir, vamos a establecer dos grupos: a) educación (pedagogía y psicopedagogía); b) psicología. Si contrastamos estos dos grupos con "denomina" (asesoramiento - consulta), se obtiene la tabla 3, que se complementa con el hecho de haber cursado o no el doctorado. Son dos análisis distintos (especialidad y doctorado). Respecto a la titulación se obtiene una X2 igual a 3'46 ( $p=0$ '063). Es decir, strictu sensu no se rechaza la hipótesis nula por una centésima. Pero se observa una cierta asociación entre las dos variables. Es decir, en el grupo que utilizan "consulta" hay un porcentaje muy elevado de educación, en relación con el grupo que utiliza asesoramiento que es más de psicología.

TABLA 3. Denominación por especialidad y doctorado

\begin{tabular}{|l|c|c|c|c|}
\hline & \multicolumn{2}{|c|}{ Titulación } & \multicolumn{2}{c|}{ Doctorado } \\
\hline & Educación & Psicología & No Doctor & Doctor \\
\hline Asesoramiento & 113 & 69 & 161 & 32 \\
\hline Consulta & 21 & 5 & 14 & 13 \\
\hline Total & 134 & 74 & 175 & 45 \\
\hline
\end{tabular}


Si nos centramos en el título de doctor y lo contrastamos con los dos grupos de "denomina" (asesoramiento - consulta), se obtiene una $x 2$ igual a 14'5 $(p<0$ '000). Es decir, hay una asociación entre el ser doctor y utilizar "consulta". Es decir, en el grupo que utilizan "consulta" hay un porcentaje muy elevado de doctores (48'1 \%) en relación con el total; mientras que de los no doctores, la inmensa mayoría (83'4 \%) utiliza asesoramiento.

Estos datos tienen que complementarse con una asociación entre el hecho de ser doctor y la especialidad (educación). La tabla 4 analiza estos datos, de donde se deriva una X2 igual a 8'96 ( $p$ $<0$ '003).

TABLA 4. Asociación entre doctorado y especialidad

\begin{tabular}{|l|c|c|c|}
\hline & Educación & Psicología & Total \\
\hline No doctor & $98\left(58^{\prime} 0 \%\right)$ & $71\left(42^{\prime} 0 \%\right)$ & $169(100 \%)$ \\
\hline Doctor & $37\left(82^{\prime} 2 \%\right)$ & $8\left(17^{\prime} 8 \%\right)$ & $45(100 \%)$ \\
\hline Total & $135\left(63^{\prime} 1 \%\right)$ & $79\left(36^{\prime} 9 \%\right)$ & $214(100 \%)$ \\
\hline
\end{tabular}

Además de los 27 que utilizan consulta, hay 16 que son profesores de universidad. De los 27 solamente hay 5 con formación en psicología, de los cuales, uno es profesor de universidad en orientación.

De la muestra de participantes que han respondido, hay profesionales de la orientación y profesores de universidad. Pero estos últimos son de educación, no de psicología.

Como en la realidad esto no tiene porque ser así en la población real, hay que interpretar que dentro de la muestra, hay un reducido grupo de profesores de universidad, que son de educación, con un alto nivel de especialización, que lee en inglés, con un doctorado, y por lo tanto están al corriente de lo que se expone en este artículo. Pero no hay en la muestra un grupo similar procedente de psicología. Esto se podría interpretar como un sesgo en la muestra. Pero por lo que indican los resultados, si hubiera un grupo similar de profesores de psicología, los resultados todavía estarían más en la dirección de utilizar el término asesoramiento.

Lo que antecede aconseja la aplicación de un análisis discriminante. Los resultados mediante el paquete SPSS permiten comprobar cómo la variable doctor y artículos en inglés son altamente significativos $(p<.000)$ en cuando a poder de discriminación. También es significativo "libros en inglés" $(p=.048)$.

La tabla 5 contiene el resumen del análisis discriminante, donde se puede observar que la probabilidad de acierto en la clasificación es del $82 ' 4 \%$.

TABLA 5. Resultados de la clasificación del análisis discriminante

\begin{tabular}{|l|c|c|c|}
\hline & \multicolumn{2}{|c|}{ Predicción del grupo } & \multirow{2}{*}{ Total } \\
\cline { 1 - 3 } & Asesoramiento & Consulta & \\
\hline Asesoramiento & $172\left(88^{\prime} 7 \%\right)$ & $22\left(11^{\prime} 3 \%\right)$ & 194 \\
\hline Consulta & $17\left(63^{\prime} 0 \%\right)$ & $10\left(37^{\prime} 0 \%\right)$ & 27 \\
\hline Casos no agrupados & $6\left(85^{\prime} 7 \%\right)$ & $1\left(14{ }^{\prime} 3 \%\right)$ & 7 \\
\hline Total & 195 & 33 & 228 \\
\hline \multicolumn{2}{|r|}{$82^{\prime} 4 \%$ de casos originales han sido clasificados correctamente } \\
\hline
\end{tabular}

Este análisis permite establecer un perfil de persona que utiliza consulta, cuyas características 
son las siguientes: se trata de un grupo reducido de personas, que en la muestra es el 11'84 \%, según se detalla en la tabla 2; leen más obras especializadas en inglés sobre orientación, tanto libros como revistas (tabla 6); son preferentemente de estudios de educación (pedagogía y psicopedagogía); muchos de ellos han cursado un doctorado (Tabla 3); y de los 27 que utilizan consulta, hay 16 que son profesores de universidad.

TABLA 6. Características diferenciales (en porcentajes)

\begin{tabular}{|l|c|c|c|c|}
\hline & Asesoramiento & Consulta & $\mathbf{t}$ & $\mathbf{p}$ \\
\hline Edad (media) & $44^{\prime} 84$ & $45^{\prime} 93$ & $0^{\prime} 546$ & .586 \\
\hline Años experiencia & $12^{\prime} 20$ & $13^{\prime} 77$ & $0^{\prime} 836$ & .404 \\
\hline Libros consultados & $6^{\prime} 86$ & $10^{\prime} 15$ & $1^{\prime} 128$ & .200 \\
\hline Artículos & $12^{\prime} 26$ & $12^{\prime} 78$ & $0^{\prime} 141$ & .888 \\
\hline Libros en ingles & $0^{\prime} 55$ & $1^{\prime} 52$ & $2^{\prime} 024$ & .044 \\
\hline Artículos en inglés & $1^{\prime} 31$ & $4^{\prime} 74$ & $4^{\prime} 088$ & .000 \\
\hline
\end{tabular}

\section{Conclusiones}

La palabra consulta, y en concreto consulta colaborativa, tienen una larga tradición en orientación. Es, sin duda, el modelo de intervención que conviene potenciar en la práctica educativa y orientadora y así se refleja en la literatura especializada.

En muchas publicaciones en castellano, se utiliza asesoramiento en lugar de consulta. Lo cual plantea un problema de duplicidad terminológica para referirse al mismo concepto. Para evitar la proliferación de la torre de Babel en orientación, convendría llegar a un acuerdo sobre la terminología a utilizar. ¿Consulta o asesoramiento?, ¿cuál de los dos conviene utilizar? Esta es la pregunta que nos hemos formulado.

Después de una revisión bibliográfica sobre el tema, se ha aplicado un cuestionario a una muestra de 228 participantes, profesionales y expertos en orientación. Se ha aplicado estadística descriptiva y un análisis discriminante. Los resultados se pueden sintetizar en las siguientes conclusiones.

Consulta está en consonancia con la tradición y permite utilizar términos de uso internacional. Pero solamente un sector reducido de profesorado universitario de pedagogía, que leen habitualmente en inglés obras especializadas, utiliza este vocablo.

Los resultados de la encuesta señalan claramente que la inmensa mayoría de profesionales de la orientación utiliza asesoramiento para referirse a lo mismo.

Conviene señalar las limitaciones de este trabajo, ya sean por el tamaño y la selección de la muestra, así como el instrumento de recogida de datos. Sin embargo, la evidencia de los resultados permite entrever el estado de la cuestión con bastante claridad.

Los datos permiten tomar decisiones respecto al uso de asesoramiento. Fijémonos que esto nos sitúa en tener que elegir a favor de la terminología utilizada por la mayoría de los profesionales de la orientación frente a la terminología de las revistas de impacto. 


\section{Referencias bibliográficas}

Álvarez González, M. (1995). Orientación profesional. Barcelona. Cedecs.

Álvarez González, M. y Bisquerra, R. (coords.) (1996). Manual de orientación y tutoría. Barcelona. Cisspraxis.

Aubrey, C. (1990). Consultation and change in education. Londres: Falmer Pres.

Bassedas, E. (1988). El asesoramiento psicopedagógico: una perspectiva constructivista. Cuadernos de Pedagogía, 159, 65-71.

Bisquerra, R. (1990). Orientación psicopedagógica para la prevención y el desarrollo. Barcelona: Boixareu Universitaria.

Bisquerra, R. (1996). Orígenes y desarrollo de la orientación psicopedagógica. Madrid. Narcea.

Bisquerra, R. (coord.) (1998). Modelos de orientación e intervención psicopedagógica. Barcelona. Cisspraxis.

Bisquerra, R. y Álvarez González, M. (1998). Modelos de orientación. En R. Bisquerra (coord.). Modelos de orientación e intervención psicopedagógica (págs. 55-65). Barcelona. Cisspraxis.

Bisquerra, R. y Álvarez González, M. (2000). La consulta colaborativa. En M. Álvarez González y R. Bisquerra (coords.), Manual de orientación y tutoría (págs. 352/1- 352/10). Barcelona. Cisspraxis.

Bonals, J., y Sánchez-Cano, M. (2007). Manual de asesoramiento psicopedagógico. Barcelona: Graó.

Brown, D., Wyne, M. D., Blackburn, J. E. y Powell, C. W. (1979). Consultation: strategy for improving education. Boston: Allyn and Bacon Inc.

Caplan, G. (1970). The theory and practice of mental health consultation. Nueva York: Basic Books.

Caplan, G., y Caplan, R. (1997). Consulta y colaboración en salud mental. Barcelona: Paidós.

Cole, E., y Siegel, I. (Coords.). (1991). Effective Consultation in School Psychology. Toronto: Hogrefe Publishers.

Conoley, J. C. y Conoley, C. W. (1982). School consultation: A guide to practice and training. Nueva York: Pergamon Press.

Dinkmeyer, D. (1962). The Consultant in Elementary School Guidance. Guidance Journal, 5, 1, 4, 95-101.

Dinkmeyer, D. y Carlson, J. (1973). El consultor psicopedagógico en la escuela. Buenos Aires: Guadalupe.

Dinkmeyer, D. y Dinkmeyer, D. (1984). School counselors and consultants in primary prevention programs. Personnel and Guidance Journal, 62, 8, 464-466.

Domingo, J. (coord.). (2001). Asesoramiento al centro educativo. Barcelona: Octaedro.

Drapela, V. J. (1983). The Counselor as consultant and Supervisor. Springfield, II.: Charles C. Thomas.

Dustin, R. y Burden, C. (1972). The counselor as behavioral consultant. Elementary School Guidance and Counseling, 7, 14-19.

Erchul, W. P., y Martens, B. K. (1997). School consultation. Nueva York: Plenum. 
Escudero, J. M., y Moreno, J. M. (1992). El asesoramiento a centros educativos. Madrid: Consejería de Educación y Cultura de la Comunidad de Madrid.

Etzion, D. (1980). Consultant's Involvement and its consequences in consultant-client verbal interaction. Journal of counseling Psichology, 27, 1-8.

Faust, V. (1967). The Counselor as a Consultant to Teachers. Elementary School Guidance \& Counseling, 5, 1, 2, 112-117.

Fullmer, D. y Bernard, H. (1972). The School Counselor-Consultant. Boston: Houghton Mifflin.

Gallessich, J. y Ladogana, A. (1978). Consultation Training Program for School Counselors. Counselor Education and Supervision, 18 (2), 100-108.

Hernández Rivero, V., Santana Vega, L. E., y González, A. C. (2007). El asesoramiento de los equipos de orientación educativa y psicopedagógica (EOEP) en el contexto de los centros educativos: un estudio cualitativo. Revista de Investigación Educativa, 25, 2, 287-304.

Huguet, T. (2011). El asesoramiento a la introducción de procesos de docencia compartida. En E. Martín y J. Onrubia (coords.), Orientación educativa. Procesos de innovación y mejora de la enseñanza (pp. 143-165). Barcelona: Graó.

Idol, L. (1990). The scientific art of classroom consultation. Journal of Educational Psychology Consultation, 1, 3-22.

Idol, L., Paolucci-Whitcomb,P., y Nevin, A. (1986). Collaborative consultation. Rockville, MD: Aspen Systems.

Kahnweiler, W. M. (1979). "The school Counselor as a Consultant: A Historical Review". Personnel and Guidance Journal, 57, 370-380.

Knoff, H. M. (1985). Discipline in the schools: An inservice and consultation program for educational staffs. School Counselor, 32, 211-218.

Kurpius, D. y Brubaker, J. (1976). Psychoeducational consultation: Definitions, functions, preparations. Bloomington, IN: Indiana University.

Lago, J. R., y Onrubia, J. (2008). Asesoramiento psicopedagógico y mejora de la práctica educativa. Barcelona: Horsori.

Lago, J. R., y Onrubia, J. (2011). Un modelo de asesoramiento para la mejora de las prácticas educativas. En E. Martín y J. Onrubia (coords.), Orientación educativa. Procesos de innovación y mejora de la enseñanza (pp. 11-32). Barcelona: Graó.

Lamothe, P. (1976). Les professionnels de la Consultation et de l'Orientation en milieu scolaire: des intervenents en mutation. L'Orientation Professionnelle, 12, 33-45.

Lee, J. (1963). Is a Guidance Consultant Needed in the Elementary School. Illinois Guidance \& Personnel Association Newsletter, 56-59.

Martín, E., y Solé, I. (coords.) (2011). Orientación educativa. Modelos y estrategias de intervención. Barcelona: Graó.

Martínez Clares, P. (2002). La orientación psicopedagógica: modelos y estrategias de intervención. Madrid: EOS.

Mc Beath, M. (1980). Consulting with teachers in two areas: grief and mourning: Relaxation techniques. Personnel and Guidance Journal, 58, 473-476.

Meyers, J. (1978). Training school psychologists for a consultation role. School Psychology Digest, 7 (3), 26-31.

Michelson,D. y Davis, J. L. (1977). A consultation model of the school counselor. The school 
counselor, 25, 98-193.

Monereo, C., y Pozo, J. I. (2005). La práctica del asesoramiento educativo a examen. Barcelona: Graó.

Monereo, C., y Solé, I. (coords.) (1996). El asesoramiento psicopedagógico: una perspectiva profesional y constructivista. Madrid: Alianza.

Morrill, W. H. Oetting, E. R., y Hurst, J.C. (1974). Dimensions of Counselor Functioning. Personnel and Guidance Journal, 52, 6, 354-359.

Morrill, W. H., Hurst, J. C. y Oetting, E. R. (1980). Dimensions of Intervention for Student Development. Nueva York: John Wiley.

Nieto, J. M., y Botías, F. (2000). Los equipos de orientación educativa y psicopedagógica. El asesoramiento a centros escolares desde un análisis institucional. Barcelona: Ariel.

Pryzwansky, W. B. (1977). Collaboration or consultation: Is there a difference? Journal of Special Education, 11, 179-182.

Robinson, S.M. (1991). Collaborative Consultation. En B. Wong (Coord.), Learning about disabilities. Nueva York: Academic Press.

Rodríguez Espinar, S. (1986). Proyecto docente e investigador. Presentado al concurso de acceso a la plaza de Catedrático de la Universidad de Barcelona en el área de métodos de investigación y Diagnóstico en Educación (Orientación educativa). Barcelona: Inédito.

Rodríguez Espinar, S. (Coord), Alvarez González, M., Echeverría, B. y Marín, M.A. (1993). Teoría y Práctica de la Orientación Educativa. Barcelona: PPU.

Rodríguez Romero, M. M. (1996). El asesoramiento en educación. Málaga: Aljibe.

Santana Vega, L. E. (2003). Orientación educativa e intervención psicopedagógica. Madrid: Pirámide.

Santana Vega, L. E., y Santana Bonilla, P. (1998). El modelo de consulta/asesoramiento en orientación. Revista de investigación educativa, 16, 2, 59-77.

Sanz Oro, R. (2001). Orientación psicopedagógica y calidad educativa. Madrid: Pirámide.

Schulte, A. C., y Osborne, S. S. (2003). When assumptive worlds collide: A review of definitions of collaboration in consultation. Journal of Educational and Psychological Consultation, 14, 2, 109-138.

Shertzer, B. y Stone, S. (1972). Manual para el asesoramiento psicológico (Counseling). Buenos Aires: Paidós.

Splete, H. H. (1988). Consultation by the Counselor. En R. Hayes y R. Aubrey, New Directions for Counseling and Human Development (pp. 275-285). Denver, Colorado: Love Pub. Co.

Umansky, D. L. y Holloway, E. L. (1984). The counselor as consultant: From model to practice. The school counselor, 31, 325-338.

Walz, R. y Benjamin, L. (1978). A change agent strategy for counselors functioning as consultans. Personnel and Guidance Journal, 56, 331-334.

West, J. F. (1990). The nature of consultation vs. colaboration: An interview with Walter B. Pryzwansky. The Consulting Edge, 2 (1), 1-3.

West, J. F., e Idol, L. (1987). School consultation (Part I): An interdisciplinary perspective on theory, models and research. Journal of Learning Disabilities, 20 (7), 388-408.

West, J. F., y Cannon, G. (1988). Essential collaborative consultation competencies for regular and special educators. Journal of Learning Disabilities, 21, 56-63. 
Young, A. Y. y Borgen, W. A. (1979). Developmental consultation in implementing career education programs. Canadian Counsellor, 13, 4, 179-183.

Fecha de entrada: 21 de noviembre de 2012

Fecha de revisión: 12 de febrero de 2013

Fecha de aceptación: 13 de mayo de 2013 